Jean-Michel Durr - Nicole Roth

\title{
Towards certification of new statistics of public interest in France
}

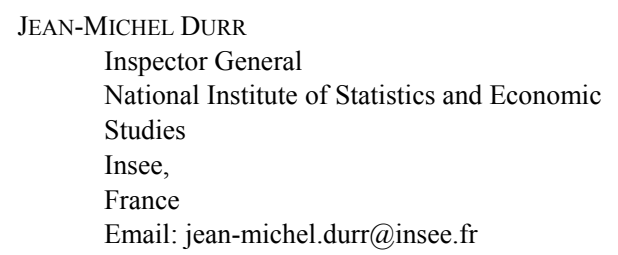

\author{
NiCOLE ROTH \\ Inspector General \\ National Institute of Statistics and Economic \\ Studies \\ Insee, \\ France \\ Email: nicole.roth@insee.fr
}

This paper presents the analysis and proposal to qualify new statistics of public interest in France. It describes the present situation regarding the qualification of statistics produced independent of the National Statistical System and the challenges related to their emergence, as these statistics may complement and sometimes challenge official statistics. The authors propose a three-tier mechanism; the first level is limited to self-documentation of a statistic based on a predefined template, the second level is the approval of such documentation, and the third level involves the approval of the documentation including the methodology and quality of output.

KEYwORDS: National Statistical System, qualification of statistics, France

Open data and economic digitalisation have increased the scope of public and private datasets and statistics. The production of data or statistics has thus become generalised to new actors who build their data sources through their activities or who reuse public sources through the growing supply of open (raw) data. On several occasions, these new statistics intersect with public statistics, providing additional insights or causing disturbances if the messages contradict, and uncover new fields of observation.

This phenomenon is not new: several surveys have been carried out by professional bodies that produced and published statistics and widely reported them in the media. What has changed is that the digital revolution has generalised the capacity of the actors of private and civil society to produce multitudes of data and extract new statistics of general interest. For example, some private companies produce reports

Hungarian StATISTICAL REVIEW, VOLUME 3, NuMBER 2, PP. 3-11. DOI: 10.35618/hsr2020.02.en003 
on employment as a by-product of their payroll management services or use webscraping techniques to collect press clippings on layoffs, hiring plans and local investments. Their underlying business model for this activity is not to commercialise aggregated statistics but to seek visibility and credibility from the media to sell commercial products, such as detailed information, to private clients.

However, the public sector is the primary producer of non-official statistics. This includes various observatories (research centres) financed by public funds or local authorities' funds (in regions or large cities). They produce statistics that can complement public statistics on a specific domain (either sectoral or geographical).

The statistical system has seized the opportunities offered by new public or private sources, rather than ignoring them and sticking to the 'business as usual' concept, which would entail a clear risk of marginalisation, as reputation and trust in the statistical system cannot replace the quest for innovation (Ricciato et al. [2018]). Several national statistical institutes (NSIs) have developed plans to use administrative data or big data as input for their statistical production. The Insee (Institut national de la statistique et des études économiques - French National Institute of Statistics and Economic Studies) established an innovation lab (the SSPLab) in May 2018, as a resource and animation centre for applied research and experimental development to promote innovation and the use of new data sources, technologies and data science methods. The SSPLab works with the Eurostat Task Force on Big Data. For example, the SSPLab has experimented the use of mobile phone data to estimate the present population figures.

Thus, national statistical systems (NSSs) have reacted quickly to invest in the field of big data and develop their use to produce official statistics. However, it cannot be ruled out that private statistics derived from new technologies challenge official statistical production. Private statistics could also complement official statistics, the issue being to acknowledge them as credible information sources, among others.

\section{Overview of the governance of official statistics in France}

Official statistics in France include statistical outputs of statistical surveys and administrative sources, produced primarily by the NSS, and in some cases by public institutions or private bodies entrusted with public service missions. Therefore, it is potentially a broad set of statistics, which constitute a public good, made available to all potential users.

The statistical system in France is governed by two bodies, the ASP (L'Autorité de la statistique publique - Official Statistical Authority)

Hungarian StatistiCAL REVIEW, VOlume 3, NumBER 2, PP. 3-11. DOI: 10.35618/hsr2020.02.en003 
and the CNIS (Le Conseil national de l'information statistique - National Council for Statistical Information). The ASP ensures that French official statistics meet the requirements of the European Statistics Code of Practice (ESCoP), as the European Statistical Governance Advisory Board does at the European level. The CNIS ensures cooperation between the producers and users of official statistics to identify the needs of actors in the social and economic fields, like the European Statistical Advisory Committee for the European Statistical System. Both the ASP and the CNIS rely on a technical committee, the Official Statistics Quality Label Committee to check quality standards (Christine-Roth [2020]).

The ASP addresses the need to qualify the statistics that underlie the public debate, and which are produced 'outside' the SS (Service statistique public Public Statistical System). Part of the statistical outputs of organisations entrusted with a public service mission is thus 'labelled' by the ASP. This procedure ensures the adherence to the ESCoP: professional independence in the design, production and dissemination of public statistics as well as the principles of objectivity, impartiality, relevance and quality of the indicators produced.

Other statistics, produced by private actors or civil society organisations, are 'calibrated' to the terms adopted by the CNIS. However, this procedure is cumbersome: it includes the assessment of the documentation and the quality assessment of the data. Therefore, the number of 'calibrated' statistics remains extremely low compared to the data produced by the private sector or civil society organisations. Nevertheless, some of them have received significant attention in the media or public debates.

Insee, in its strategy for 2025, aims at 'placing the statistical service as a data certifier' to complement its statistical production. This approach seems legitimate, in a context where the phenomenon of 'fake news' is growing rapidly. However, questions arise: how to develop a qualification function for these new statistics to give more visibility to those that bring added value? What would be the relevant field to address? What are the possible methods for implementing this new function?

\section{Issues and challenges}

The first issue is to provide relevant information to the public by accompanying statistics produced with corresponding metadata, making it useful and usable. The first and most fundamental purpose of statistical metadata is to help users interpret, understand and analyse statistics. The level of detail of metadata depends on the needs and skills of users. For example, a journalist may not have the time to digest a

HUNGARIAN StaTISTICAL REVIEW, VOLUME 3, NUMBER 2, PP. 3-11. DOI: 10.35618/hsr2020.02.en003 
large volume of detailed metadata and will need minimal information to avoid misinterpretation. Conversely, a social scientist may even want to question the assumptions made by the original producer of statistics and deduce new statistical results based on alternative hypotheses. The latter type of user should have access to all relevant assumptions and other circumstances in the collection, preparation and estimation of process data, as designed and operated by the statistical producer (Dippo-Sundgren [2000]).

Beyond metadata, the quality of data and statistics is also important. The ESCoP governs the production of the public statistical system in France. It ensures that statistics are based on the principles of quality, including professional independence, relevance, quality of processes and products, and are accompanied by corresponding metadata.

Statistics produced outside the NSS do not always provide the necessary metadata and do not comply with quality commitments. Private companies present some elements of methodology description, but often incompletely. For example, the field covered or possible fluctuations in the collection of information that may affect temporal comparability, are often missing. Even the concept and process of measurement may be incomplete, leading to misinterpretation.

Statistical literacy is, therefore, of paramount importance for users to be able to understand how statistics are produced and what their quality is. For example, Radermacher [2018] argues that statisticians should pro-actively invest in developing 'datacy' capabilities in society to educate citizens to make better use of statistics, official or otherwise.

\section{Proposed mechanism}

The phenomenon of fake news is becoming increasingly important, and journalists are confronted daily with the need to verify the information available to them. In the French media, as elsewhere in the world, several newsrooms have now columnists dedicated to fact-checking. The basis of journalism is to cross-check sources and control their reliability (Léchenet [2015]). Thus, journalists conduct their research by themselves rather than be guided by a stakeholder. Therefore, NSIs must be careful in 'qualifying' statistics produced outside the NSS, with the risk of being accused of protecting their monopoly using a 'name and shame' mechanism.

The only mechanism to qualify unofficial statistics was found in the UK (United Kingdom). The ORS (Office for Statistics Regulation) of the UK Statistics Authority has developed common standards, based on the three pillars of the UK Code of

Hungarian Statistical ReVIEW, Volume 3, NumBER 2, PP. 3-11. DOI: 10.35618/hsr2020.02.en003 
Practice for Statistics (UK Statistics Authority [2018a]): trustworthiness, quality and value that can also apply to unofficial statistics. The ORS proposes to producers of unofficial statistics to voluntary commit to these standards by issuing a 'voluntary application statement' (UK Statistics Authority [2018b]). The statements are then published on the ORS website. However, in the absence of a standard framework, the statements are heterogeneous. The ORS hopes that this approach will raise interest and create a community. The first volunteers are more in the public or para-public domain, like Greater London, but some large companies have declared themselves ready to join the process. The UK Parliament has shown interest in the Code of Practice and its extension voluntarily. For companies, the motivation to subscribe to the voluntary declaration is a concern of reputation.

It is important to make a distinction between data and statistics. Data are the raw material for building statistics, according to predefined concepts (Desrosières [1998]). Therefore, the mechanism proposed by Insee aims at qualifying statistics, and not sources of data, following a three-tier process of qualification with a growing requirement gradient.

Three-tier mechanism for the qualification of statistics

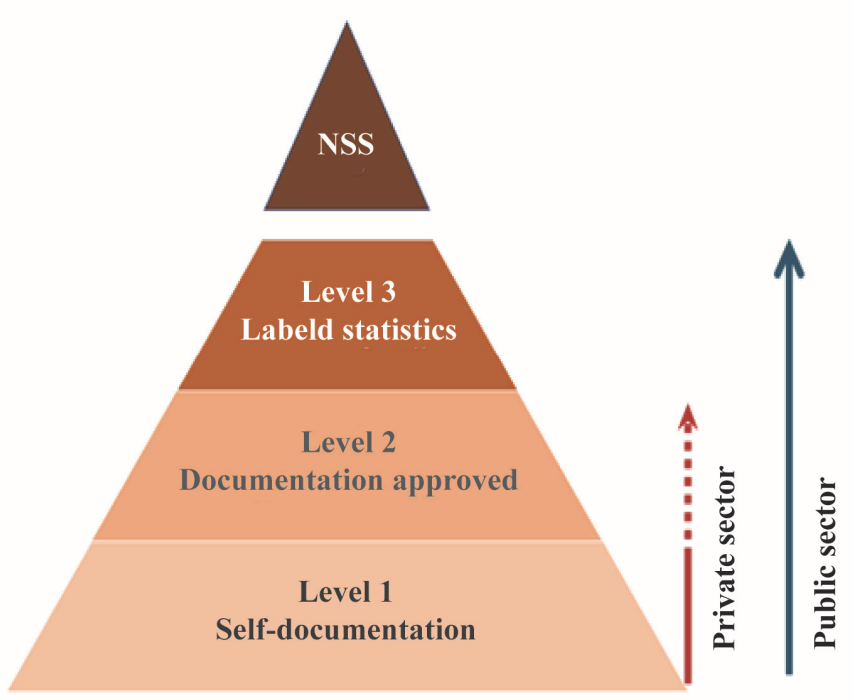

The first level requires self-documenting statistics according to a standardised documentation template. The second qualification level includes the approval (certification) of the documentation, for volunteering producers. This does not mean the approval of the methodology or outputs, just that the documentation is correctly filled out to provide the necessary basic information to users. This procedure primarily concerns

Hungarian Statistical ReVIEW, VOLUME 3, NuMBER 2, PP. 3-11. DOI: 10.35618/hsr2020.02.en003 
producers of statistics entrusted with public service missions, potentially far more likely to adhere to such a scheme than private producers. The assumption is that this could positively create a ripple effect, even in the private sphere. The third level of qualification corresponds to the labelling of official statistics: it is therefore reserved for statistics produced by organisations fulfilling public service missions. It currently involves an assessment of the relevance of the output and the methodology used, and the verification of compliance with the main principles of the ESCoP. As Insee and the statistical departments of ministries, which form the NSS, are connected to several partners producing public statistics, members of the NSS could contribute significantly to the labelling of their partners' sources. This would create a bottom-up approach, leading to extending the scope of labelled statistics.

\subsection{A standard for documenting statistics}

This approach involves the proposal of a standard documentation template based on the metadata description of European statistics, the single integrated metadata structure (SIMS) and inspired by its principles. The documentation standard is less demanding than that required to produce official statistics. It constitutes a subset of the rules of the official statistics, which materialise through the ESCoP. This new certification service would be available to organisations performing public service missions or voluntarily producing data of general interest. For example, the current health crisis highlights the value of using statistics derived from private data sources, provided that their scope and methods are transparent.

The documentation template describes the statistics and how they are produced:

- What is the legal or regulatory context or any other agreement entrusting responsibility for the development and dissemination of these statistics?

- On which classifications are they based?

- What is the level of detail of the published data?

- How often is the data published?

- What types of indicators are calculated?

- How are these indicators produced? (general public description)

- Are they understandable by a non-expert user? (accessibility of metadata)

For non-official statistics producers, the first level involves self-documenting the statistics according to the proposed template and making it available on their

HUNGaRian StatisTical ReVIEW, VOLUME 3, NUMBER 2, PP. 3-11. DOI: 10.35618/hsr2020.02.en003 
website. Any producer of statistics, even from the private sector, can use the standard documentation template without seeking official approval.

\subsection{Certification procedure}

The second qualification level consists of the formal approval (certification) of this standard documentation. The certification process aims to qualify a minimum level of documentation of a statistic, to ensure its transparency and allow the public to understand how it was developed and how to use it. This does not mean approval of the methodology or outputs, but the recognition that the organisation produces documentation per the expected standard and thus ensures sufficient transparency to allow its appropriation by the users. The approval also aims to develop a culture of statistical literacy or 'datacy' for users.

The documentation approval process is divided into two stages. The first stage involves examining the eligibility of the request for a certification by the CNIS. For example, statistics certified must be of general interest, published regularly and be widely disseminated statistics, without limitation of access, and provide information of public interest.

The second stage is the certification of the documentation, pronounced by the CNIS and based on the technical instructions of the Official Statistics Quality Label Committee. The documentation is first reviewed by experts mobilised by this committee and then published for certified statistics on the CNIS website. Publishing of negative decisions is avoided. The certification of the documentation is granted for a limited, renewable period, and an online repository describes the list of approved statistics.

\subsection{Labelling procedure}

The third level implies an assessment of the quality and relevance of the output. The French Official Statistics Authority has defined another procedure, more complete than the certification of documentation, awarding a statistical quality label. Public administrations and bodies governed by private law exercising a public service mission, are eligible for this label. To obtain the label, they must not only comply with the documentation standard but also with several additional criteria. These additional criteria aim to ensure the methodological quality of the statistics produced, the reliability of their production process and to guarantee the conditions of objectivity and independence of their distribution.

The labelling procedure for a statistic is, therefore, more demanding than the certification procedure. The authority responsible for labelling is the ASP.

HUNGARIAN StaTISTICAL REVIEW, VOLUME 3, NUMBER 2, PP. 3-11. DOI: 10.35618/hsr2020.02.en003 
It examines the documentation and four additional dimensions, based on the ESCoP, with the support of the Official Statistics Quality Label Committee. The four dimensions include 1 . governance of the production and dissemination of statistics, for neutrality and objectivity; 2. the conditions for disseminating statistics, and access to researchers, ensuring respect for statistical confidentiality; 3 . the robustness of the methodology and the reliability of the production process; and 4. the relevance of statistics, considering the needs of users. The first dimension concerns the guarantee provided by the requesting service to govern its statistical production. The factual elements to appreciate the added value for users of these statistics are the organisation and the resources devoted to the production of statistics, overall coordination with the NSS through reference classifications, and sharing of methods. The second dimension relates to the dissemination of statistics, which must be distinct from the institutional communication of the organisation, particularly by announcing its release calendar in advance. The accessibility of the series and the quality of the metadata made available to users constitutes an additional element of the assessment, with the objective of transparency and readability of statistics produced. Another criterion concerns researchers' accessibility to individual data from statistical databases, in a secure environment respecting statistical confidentiality. The third dimension refers to the quality of the statistical production that undergoes sound methodology and is precisely documented to be audited by experts in the field, by explaining the actions taken to control the risks of any nature during the production process. Other criteria relate to temporal comparability and the consistency or articulation of the statistics produced by the SSP. Finally, the fourth dimension aims to assess the relevance of statistics for users: do they meet the main knowledge needs, do they inform public decision-making? This relevance can be objectified by regular consultation with users and considering, as far as possible, their requests, leading to periodic reviews in the process, or innovations to improve the offer based on the changing needs.

The labelling instruction takes place in practice in a single step combining the documentary examination and product quality review. It is carried out by the Official Statistics Quality Label Committee and sent for approval to the ASP. The ASP makes its decision public in the Official Gazette.

\section{Conclusion}

In contrast to the above, the approval procedure would remain inoperative if potential candidates are not aware of it and if users do not differentiate between different levels of quality. The pyramid is made up of increasing levels of quality

Hungarian Statistical Review, Volume 3, NumBer 2, PP. 3-11. DOI: 10.35618/hsr2020.02.en003 
requirements with the NSS at the top, and its commitment to comply with the ESCoP must be clearly presented.

Finally, one of the ways to control or limit the audience of statistics that does not respect the quality standards is to communicate more convincingly on the commitments and achievements concerning the quality of the NSS. Therefore, it is useful to develop more user-oriented communication on statistical quality, on the Insee website, following the example of the UK Statistics Authority that has organised its Code of Practice for statistics into three pillars (trustworthiness, quality, and value) so that users become aware of these issues.

\section{References}

Christine, M. - Roth, N. [2020]: Le Comité du label: un acteur de la gouvernance au service de la qualité des statistiques publiques (The Official Statistics Quality Label Committee: An actor in governance serving the quality of public statistics). Courrier des statistiques N5 (to be published). Insee. Paris.

Desrosières, A. [1998]: The Politics of Large Numbers - A History of Statistical Reasoning. Harvard University Press. Cambridge.

Dippo, C. - Sundgren, B. [2000]: The Role of Metadata in Statistics. Paper presented at the International Conference on Establishment Surveys II. 17-21 June. Buffalo. https://ww2.amstat.org/meetings/ices/2000/proceedings/S57.pdf

LeChenet, A. [2015]: Le journalisme de données. Synthèse des débats. Cafés de la statistique. Soirée du 14 avril. Société Française de statistique. Paris.

RADERMACHER, W. [2018]: Official statistics in the era of big data opportunities and threats. International Journal of Data Science and Analytics. Vol. 6. 26 April. pp. 225-231. https://doi.org/10.1007/s41060-018-0124-z

Ricciato, R. - SkAliotis, M. - Wirthmann, A. - GiannAKouRis, K. [2018]: Towards a Reference Architecture for Trusted Smart Statistics. Eurostat Task Force on Big Data. Paper presented at DGINS 2018. 10-11 October. Bucharest.

UK Statistics Authority [2018a]: Code of Practice for Statistics, Edition 2.0. February. https://code.statisticsauthority.gov.uk/wp-content/uploads/2018/02/Code-of-Practice-forStatistics.pdf

UK Statistics Authority [2018b]: Building Confidence in Statistics: Voluntarily Committing to Trustworthiness, Quality and Value. Edition 1.0. May. https://code.statisticsauthority. gov.uk/wp-content/uploads/2020/07/Guide-to-Voluntary-Application.pdf

Hungarian StatisticAl REVIEW, VOLUME 3, NuMBER 2, PP. 3-11. DOI: 10.35618/hsr2020.02.en003 